\title{
BENCHTOP POLYMER MEMS AS A LOW-COST EDUCATIONAL TOOL
}

\author{
Mario Urdaneta, Remi Delille, Elisabeth Smela \\ Mechanical Engineering Department, University of Maryland \\ College Park, Maryland, USA
}

As microsystems become ubiquitous and nanotechnology increases in importance, it becomes ever more imperative to include these topics in engineering curricula. One of the most effective ways to teach microfabrication methods is through hands-on laboratories in clean rooms since MEMS structures are manufactured using a unique set of techniques, and students do not have an intuitive grasp of the underlying concepts. Most educational institutions, however, do not have the facilities required to offer such labs, so that MEMS courses are limited to lectures. It would therefore be useful if there were meso-scale lab demonstrations that could be done in standard laboratories, or even in classrooms, to convey the basic concepts, such as masking, sacrificial layers, alignment, and so forth.

We present the concept of a MEMS kit with associated lesson plans that can be used to give students hands-on fabrication experience that is analogous to what is done in a clean room, but on a larger scale so that no expensive equipment is needed. The photoresist analog is a photopatternable adhesive that can be used in room light and exposed through transparency masks with a handheld UV lamp. Among the concepts that can be demonstrated are photolithography (exposure, alignment, developing), etching (Figure 2), sacrificial layers, lift-off, shadow masking, electroplating in templates ("poor man's LIGA"), micromolding of PDMS, microstamping, and laminar flow in microchannels (see Figure 3). Devices that can be fabricated include single and multi-level microchannels, check valves, and bilayer thermal actuators. The supplies that are required for the photolithography are shown in Figure 1, and their total cost is less than $\$ 500$. Below, we outline a sample lesson plan for a laminar flow device.

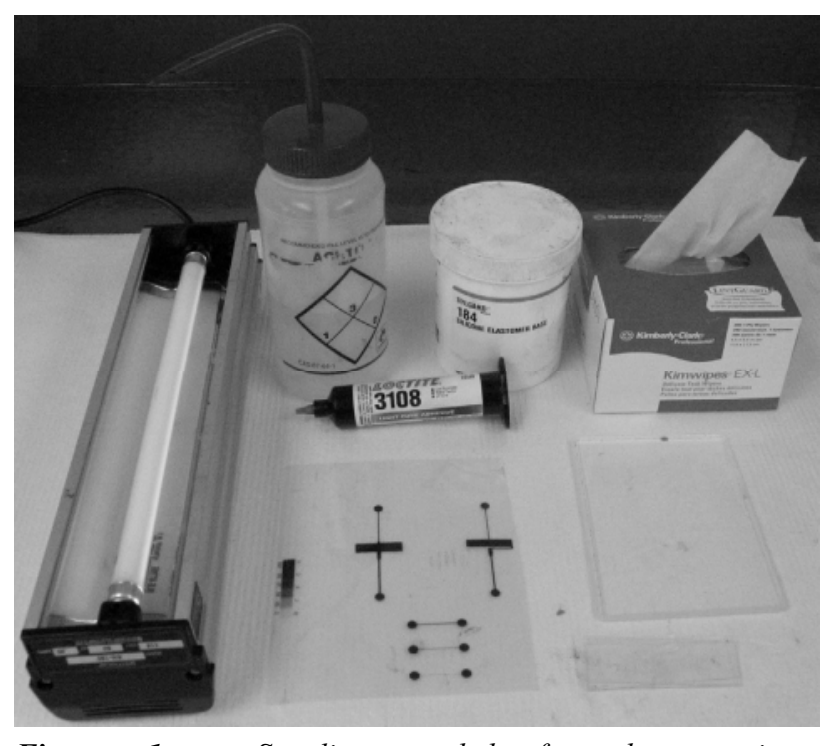

Figure 1. Supplies needed for demonstrating photolithography include a hand-held UV lamp, Loctite adhesive, transparency masks, paper tissue, PDMS elastomer base, ethyl acetate or acetone, glass slides, and a sheet of acrylic.

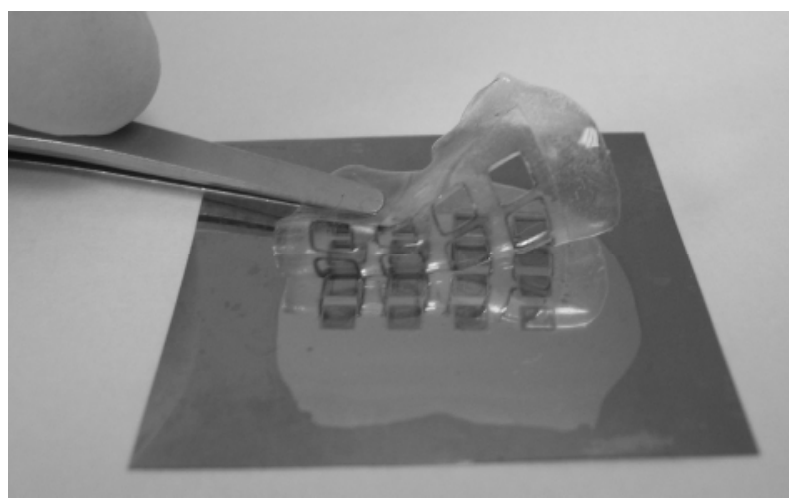

Figure 2. An etch mask being removed from a metal-coated Kapton film after wet etching. Such concepts can be demonstrated in a classroom environment using minimal infrastructure.

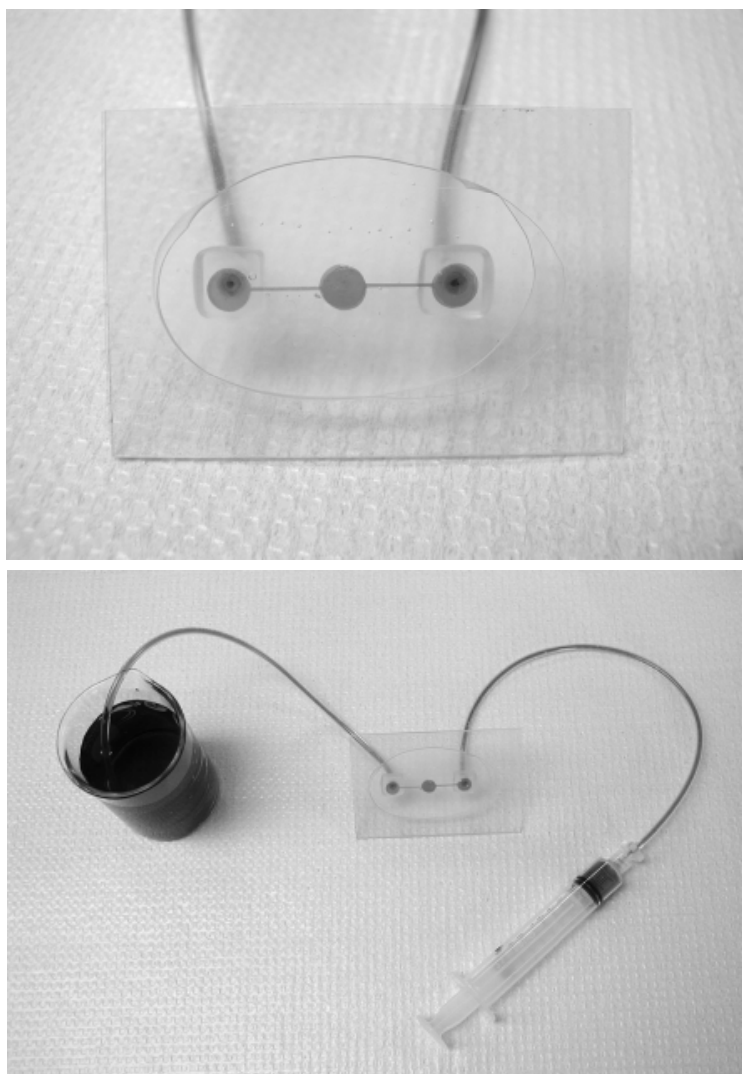

Figure 3. Two-level microfluidic devices such as this can be fabricated. 


\section{SAMPLE LESSON PLAN: FABRICATION OF A LAMINAR FLOW DEVICE}

This demonstration is based on the 1994 paper by Branebjerg et al.: J. Branebjerg, B. Fabius, P. Gravesen, "Application of Miniature Analyzers from Microfluidic Components to $\mu$ TAS," A. van den Berg and P. Bergveld [eds.], Proceedings of Micro Total Analysis Systems Conference, Twente, the Netherlands, Nov. 21-22, pp. 141$151,1994$.

\section{Background}

\section{a. Microfluidics}

Microfluidics refers to the manipulation of fluids at the microscale. The manipulations include chemical mixing (e.g. for drug synthesis), DNA or protein separation (for example by electrophoresis), and cell sorting. One emerging application for microfluidics is $\mu \mathrm{TAS}$, or micro total analysis systems, which seek to integrate entire laboratories onto a microchip... [truncated in the interest of space]

\section{b. Laminar Flow}

Fluid mechanics at the microscale is greatly affected by scaling laws: viscous forces become large while inertial forces all but disappear and the surface-area-to-volume ratio increases, making diffusion an important transport phenomenon. The ratio between the inertial and the viscous forces is given by the Reynold's number: $R e=D_{h} \rho u / \mu$, where $D_{h}$ is the channel's hydraulic diameter, $\rho$ is the fluid density, $\mathrm{u}$ is the velocity, and $\mu$ is the dynamic viscosity. Whether flow is laminar (the local velocity vector points downstream) or turbulent depends on the Reynold's number. In microfluidics fluid flow is almost always laminar because the channel is so small. This makes it impossible for fluids to mix through convection...

For more information about flow properties at small scales:

-E.M. Purcell, "Life at low Reynold's numbers", American Journal of Physics vol 45, pages 3-11, 1977.

For more information on laminar flow:

-R. Fox, A. McDonald, P. Pritchard, Introduction to Fluid Mechanics, Wiley, 2003.

\section{c. Diffusion}

Diffusion is the movement of chemical species down a concentration gradient...

Species will diffuse while they are being carried by a flow. Species in an infinitely thin fluid element will spread out to a width of approximately $\sqrt{D \tau}$ during a time $\tau$, where $\mathrm{D}$ is the diffusivity...

For more on diffusion:

-S. Senturia, Microsystem Design, Kluwer Academics Publishers, 2001

\section{Pre-Lab Discussion}

1. Given that the diffusion coefficient for most molecules in water is on the order of $10^{-5} \mathrm{~cm}^{2} / \mathrm{sec}$, and given a channel cross section of $300 \mu \mathrm{m} \times 100 \mu \mathrm{m}$, how long must the channel be for two fluids colored yellow and blue to mix if they are fed into this channel from a Y-junction such as the one shown in step 4?

2. Sketch what you think will happen over a $3 \mathrm{~cm}$ long channel.

3. ...

\section{Material List}

3" by 2" glass slide with four pre-drilled 1/16" diameter holes

3 " by 2 " glass slide with no holes

1 dual syringe

4 plastic tubes and connectors

Loctite 3108 elastomer precursor

transparency mask \#3 (cut to 3" by 2")

yellow and blue food coloring

water

magnifying glass

Spectroline EN-180 UV lamp (center wavelength 365 nm)

acetone

transparency strips (cut to 2.75 " by 0.25 ")

transparency cover (cut to 3 " by 2")

\section{Fabrication Instructions}

Step 1. Insert connectors into the four port holes, then surround the connectors with a small amount of Loctite $3108(\sim 0.5$ $\mathrm{mL}$ ) and expose to ultraviolet light for 30 seconds to glue the connectors in place and prevent leakage.

Step 2. Dispense approx. $2 \mathrm{~mL}$ of Loctite 3108 onto the center of the glass substrate.

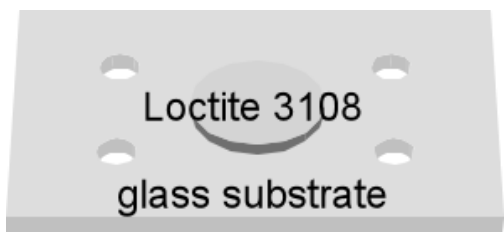

Step 3. Place the two spacers (transparency strips, $100 \mu \mathrm{m}$ thick) on the outside of the four port holes. These will determine the thickness of the polymer layer (i.e. the height of the microchannel).

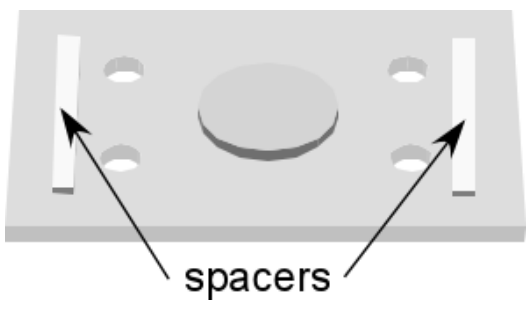


